TU-888

IPMU11-0134

UT-11-29

\title{
Higgs mass and inflation
}

\author{
Kazunori Nakayama $^{a}$ and Fuminobu Takahashi ${ }^{b, c}$ \\ ${ }^{a}$ Department of Physics, University of Tokyo, Tokyo 113-0033, Japan \\ ${ }^{b}$ Department of Physics, Tohoku University, Sendai 980-8578, Japan \\ ${ }^{c}$ Institute for the Physics and Mathematics of the universe, University of Tokyo, \\ Kashiwa 277-8568, Japan
}

\begin{abstract}
We show that the standard-model Higgs boson mass $m_{h}$ is correlated with the spectral index of density perturbation $n_{s}$ in the inflation scenario with the inflaton being identified with the B-L Higgs boson. The Higgs boson mass ranges from $m_{h} \simeq 120 \mathrm{GeV}$ to $140 \mathrm{GeV}$ for $n_{s} \simeq 0.95-0.96$. In particular, as $n_{s}$ approaches to 0.96 , the Higgs mass is predicted to be in the range of $125 \mathrm{GeV}$ to $140 \mathrm{GeV}$ in the case of relatively light gauginos, and $120 \mathrm{GeV}$ to $135 \mathrm{GeV}$ in the case where all SUSY particle masses are of the same order. This will be tested soon by the LHC experiment and the Planck satellite. The relation is due to the PeV-scale supersymmetry required by the inflationary dynamics. We also comment on the cosmological implications of our scenario such as non-thermal leptogenesis and dark matter.
\end{abstract}


The inflationary paradigm [1] has been well established so far. However, despite its great success, it is not fully known how the inflation occurred, how the Universe was reheated after inflation, how the dark matter as well as the baryon asymmetry were created.

If the inflaton is a gauge singlet field with very weak couplings to the standard-model particles, it would be challenging to pin down the inflation model. Instead, let us focus on a new inflation model recently proposed by the present authors [2], in which the $\mathrm{B}-\mathrm{L}$ Higgs boson plays the role of the inflaton. The theoretical framework is the minimal extension of the standard model $(\mathrm{SM})$, namely, $\mathrm{SM}+$ right-handed neutrinos + gauged $\mathrm{U}(1)_{\mathrm{B}-\mathrm{L}}$. The small but non-zero neutrino masses can be explained beautifully by the seesaw mechanism [3], if there are heavy right-handed neutrinos. With the addition of the three right-handed neutrinos, it is reasonable to introduce the $\mathrm{U}(1)_{\mathrm{B}-\mathrm{L}}$ gauge symmetry, because it is required by the charge quantization condition and is also motivated by the GUT gauge group such as $\mathrm{SO}(10)$. Thus, we consider the framework, $\mathrm{SM}+\nu_{R}+\mathrm{U}(1)_{\mathrm{B}-\mathrm{L}}$, as the minimal extension of the SM. There are models where the GUT Higgs, including $\mathrm{U}(1)_{\mathrm{B}-\mathrm{L}}$ Higgs, is identified as the waterfall field in hybrid inflation [4]. In Ref. [2] it has been shown that the inflation model using the B-L Higgs boson works successfully if there is supersymmetry (SUSY) at a scale below $\sim 10^{3} \mathrm{TeV}=\mathrm{PeV}$. The presence of SUSY below the $\mathrm{PeV}$ scale is crucial for canceling the Coleman-Weinberg (CW) potential [5] arising from the B-L gauge boson loop 1

Since it is conceivable that the inflationary dynamics has affected the selection of our Universe in the string landscape, the SUSY breaking scale may also be determined by the inflationary selection.2 Indeed, if there is a bias toward larger SUSY breaking scale, we expect that the upper bound on the SUSY breaking scale is saturated. The precise value of the upper bound depends on the detailed structure of the inflaton potential, and so, it is related with the properties of density perturbation such as the spectral index. Since the PeV-scale SUSY gives rise to sizable radiative corrections to the Higgs mass, we can

\footnotetext{
${ }^{1}$ The possibility that the gauge non-singlet inflaton is protected from radiative corrections by SUSY was pointed out in Ref. 6.

${ }^{2}$ Considering that weak-scale SUSY is not free of fine-tunings and typically requires a fine-tuning at the percent level for the correct electroweak breaking and that the observed cosmological constant lies in the anthropic window [7, we do not rely on the conventional naturalness argument in this letter.
} 
derive a non-trivial relation between the Higgs mass $m_{h}$ and the spectral index $n_{s}$. This is the main result of this letter.

Here we summarize the results of the inflation model in Ref. [2]. We present here the non-SUSY version for simplicity, although it is possible to write down the model in a supersymmetric language 3 . Let us consider an inflaton potential given by

$$
V(\varphi)=V_{0}-\frac{\kappa}{2 n} \frac{\varphi^{2 n}}{M_{*}^{2 n-4}}+\frac{\lambda}{2 m} \frac{\varphi^{2 m}}{M_{*}^{2 m-4}}
$$

where $\kappa$ and $\lambda$ are numerical coefficients, $m$ and $n$ are integers satisfying $m>n \geq 2$ and $M_{*}$ is a cut-off scale of the theory. We expect $M_{*}$ to be not far from the GUT scale $\sim 10^{15-16} \mathrm{GeV}$. Here we have focused on the radial component of the B-L Higgs boson $\phi$

$$
\varphi \equiv \sqrt{2}|\phi|
$$

In SUSY, the inflaton actually corresponds to the D-flat direction of $\mathrm{U}(1)_{\mathrm{B}-\mathrm{L}}$. This potential has a global minimum at $\varphi=\varphi_{\min }$ given by

$$
\varphi_{\min }=\left(\frac{\kappa}{\lambda}\right)^{\frac{1}{2(m-n)}} M_{*}
$$

which gives the $\mathrm{U}(1)_{\mathrm{B}-\mathrm{L}}$ symmetry breaking scale at low energy. We fix this scale to be $10^{15} \mathrm{GeV}$ as suggested by the atmospheric neutrino oscillations and the seesaw mechanism [3].

The inflation takes place if the initial position of $\varphi$ is sufficiently close to the origin, which is expected to be the case if there is thermal plasma before the inflation. The scalar spectral index, $n_{s}$, is given by

$$
1-n_{s}=\frac{2}{1+N \frac{2 n-2}{2 n-1}},
$$

where $N$ is the e-folding number. In the limit $n \gg 1$, it approaches to $n_{s}=0.96$ for $N=50$, which is close to the center value of the WMAP result [8]. For $n=3$, the spectral index is about 0.95 , which is also consistent with observation.

\footnotetext{
${ }^{3}$ The potential (1) with $m=2 n$ can be derived from the superpotential, $W=X\left(v^{2}-k(\phi \bar{\phi})^{n}\right)$, with an appropriate Kähler potential, where $\phi$ and $\bar{\phi}$ denote a conjugate pair of superfields transforming under $\mathrm{U}(1)_{\mathrm{B}-\mathrm{L}}$. This form of the superpotential is ensured by assigning a discrete $Z_{n}$ symmetry under which $\bar{\phi}$ is charged. Details are found in Ref. [2].
} 
The reheating takes place through the coupling with the right-handed neutrinos,

$$
\mathcal{L}=-\frac{y_{N}}{2} \phi \bar{\nu}_{R}^{c} \nu_{R}+\text { h.c. }
$$

which generates large Majorana masses for $\nu_{R}$. The coupling constant $y_{N}$ is expected to be order unity for the heaviest $\nu_{R}$. The non-thermal leptogenesis [10] works successfully for $n \geq 3$ [2]. So we focus on the case of $n \geq 3$, although we include the case of $n=2$ in our analysis for completeness.

So far we have used the inflaton potential at the tree-level. The inflaton, the B-L Higgs boson, necessarily couples to the $\mathrm{U}(1)_{\mathrm{B}-\mathrm{L}}$ gauge boson. Furthermore, it is coupled to the right-handed neutrinos to generate large Majorana masses. Due to these interactions, the inflaton potential receives corrections at the one-loop level. In fact, it is well known that the $\mathrm{CW}$ potential arising from the gauge boson loop makes the effective potential so steep that the resultant density perturbation becomes much larger than the observed one [12]. One way to cancel or suppress the CW potential is to introduce SUSY. The successful inflationary dynamics requires the following inequality to be satisfied [2]:

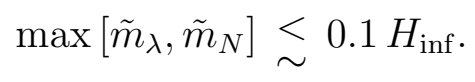

where $H_{\text {inf }}$ is the Hubble scale during inflation, $\tilde{m}_{\lambda}$ and $\tilde{m}_{N}$ represent the soft SUSY breaking masses of the $\mathrm{B}-\mathrm{L}$ gaugino and the right-handed sneutrino, respectively. We have here approximated the $\mathrm{U}(1)_{\mathrm{B}-\mathrm{L}}$ gauge coupling as well as the coupling of $\phi$ to the heaviest right-handed neutrino to be of order unity. The effect of supergravity is also negligible if a similar inequality, $m_{3 / 2} \lesssim H_{\text {inf }}$, is satisfied [2].

The inflationary scale is determined by the WMAP normalization on the density perturbation as $H_{\mathrm{inf}} \simeq 10^{6}-10^{8} \mathrm{GeV}$ depending on model parameters. Assuming that the bound (6) is saturated, soft SUSY breaking masses for the SUSY SM (SSM) particles are expected to be much heavier than the weak scale. This leads to relatively heavy lightest Higgs boson mass [13]. We have calculated the lightest Higgs boson mass along the line of Ref. [14. Fig. 1 1 shows the prediction for the Higgs mass as a function of $n$ for a different set of $\tan \beta$ defined at the scale $\tilde{m}$. We assume that the bound (6) is saturated and also that gauginos as well as higgsino masses are $1 \mathrm{TeV}$ while all other SUSY particles have masses of $\tilde{m}$. The assumption on the gaugino and higgsino masses does not much 
affect the result as long as they are $\mathcal{O}(1) \mathrm{TeV}$. We set $m=n+1, \varphi_{\min }=10^{15} \mathrm{GeV}$ and $M_{*}=10^{16} \mathrm{GeV}$. Thick (thin) lines correspond to $\tan \beta=50$ (2), and solid, dashed, dotted lines correspond to $m_{t}=175,173,171 \mathrm{GeV}$, respectively. In the bottom panel, we show the correlation between the spectral index $n_{s}$ and Higgs mass. We note that the precise values of $m$ and $M_{*}$ are not relevant, while changing the $\mathrm{B}-\mathrm{L}$ breaking scale slightly affects the results. If we take $\varphi_{\min }=10^{14} \mathrm{GeV}$, the predicted Higgs mass decreases by about $5 \mathrm{GeV}$ at large $n$. Focusing on $n \geq 3$, the Higgs mass ranges from $m_{h}=120 \mathrm{GeV}$ to $145 \mathrm{GeV}$, for $n_{s}=0.95-0.96$. It is seen that the Higgs mass saturates at around $125-145 \mathrm{GeV}$ for large $n$. Also the Higgs mass has a clear correlation with the scalar spectral index $n_{s}$. Fig. 2 shows the same plots but for the case where all SUSY particle masses are set to be the scale of $\tilde{m}$. In this case, the predicted Higgs mass is reduced : $m_{h}=115 \mathrm{GeV}$ to $140 \mathrm{GeV}$, for $n_{s}=0.95-0.96$. This is mainly because the running of the Higgs quartic coupling does not receive correction from Higgs-higgsino-gaugino couplings below the scale $\tilde{m}$.

The inflation model considered in this paper has interesting cosmological implications. First, the inflaton mainly decays into the right-handed neutrinos through (5). Thus, the non-thermal leptogenesis [9, 10, 11] works naturally. The reheating temperature ranges from $T_{R} \simeq 10^{8} \mathrm{GeV}$ to $10^{10} \mathrm{GeV}$. Secondly, the gravitino problem is avoided in our scenario. Assuming gravity or anomaly mediation, the gravitino mass $m_{3 / 2}$ is expected to be about the PeV scale, and so, its effect on cosmology is very mild. As to the nonthermal gravitino production from the inflaton [15], the branching ratio into the gravitinos are suppressed because the inflaton has a renormalizable coupling with the right-handed neutrinos.

In the case of split SUSY [13] with gauginos (especially gluino) within the reach of LHC, various types of signature may be observed [16]. In the anomaly mediation [17], the Wino is likely the lightest SUSY particle (LSP). Assuming $m_{3 / 2} \simeq 1 \mathrm{PeV}$, the Wino mass is about $3 \mathrm{TeV}$. Interestingly, the thermal relic of the Wino of mass $\sim 3 \mathrm{TeV}$ can account for the observed dark matter abundance [18]. This is an interesting coincidence: the SUSY breaking scale inferred from the inflation corresponds to the one required by the thermal relic abundance of the Wino LSP. In the gravity mediation, we expect that 

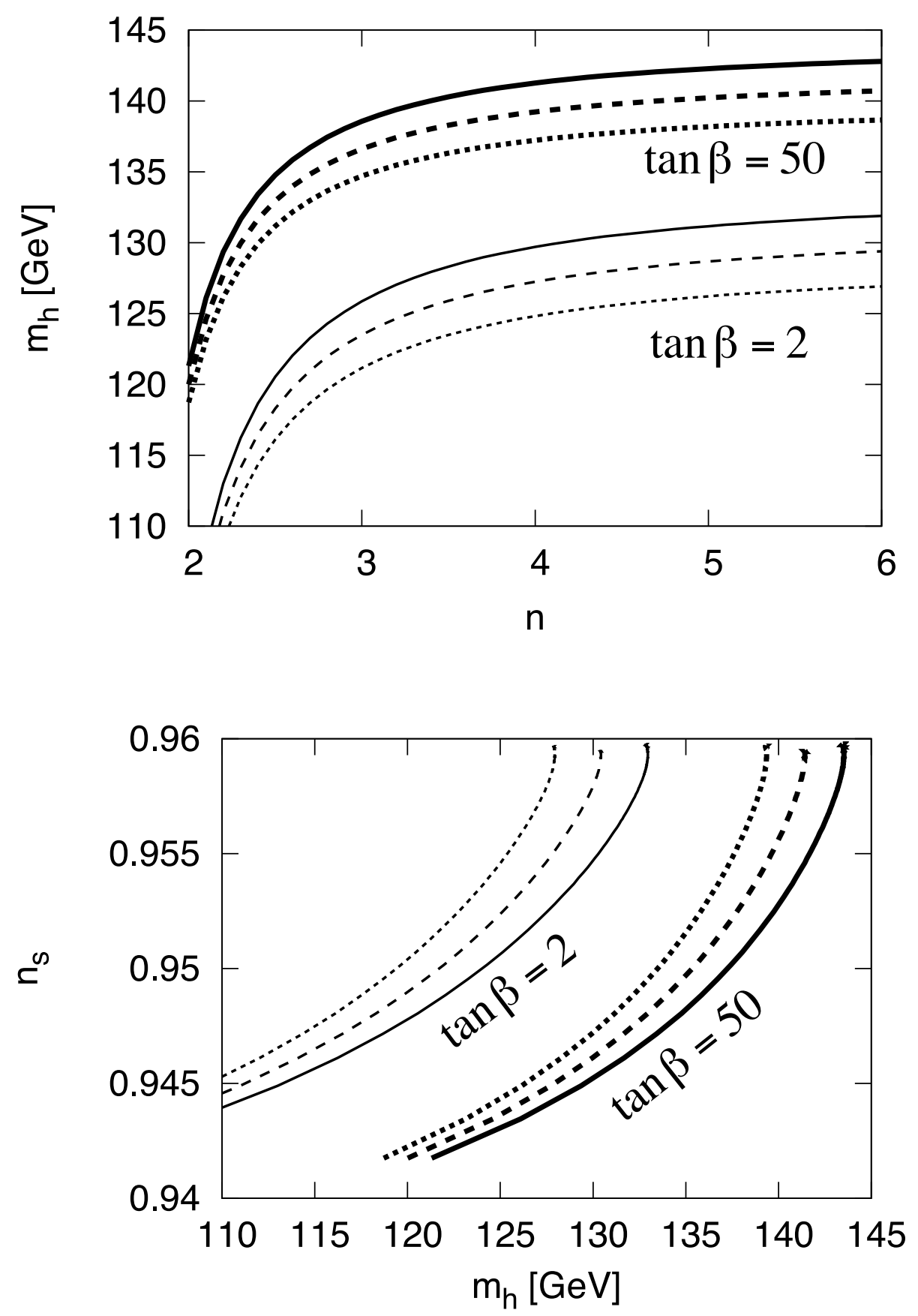

Figure 1: (Top) The prediction for Higgs mass as a function of $n$ for a different set of $\tan \beta$ and $m_{t}$ in split SUSY scenario, where gaugino and higgsino masses are set to be $1 \mathrm{TeV}$. Thick (thin) lines correspond to $\tan \beta=50$ (2), and solid, dashed, dotted lines correspond to $m_{t}=175,173,171 \mathrm{GeV}$, respectively. We set $m=n+1, \varphi_{\min }=10^{15} \mathrm{GeV}$ and $M_{*}=10^{16} \mathrm{GeV}$. The results remain almost intact if we change the values of $m$ and $M_{*}$. (Bottom) The correlation between the spectral index $n_{s}$ and Higgs mass. Meanings of each line are same as the top panel. 

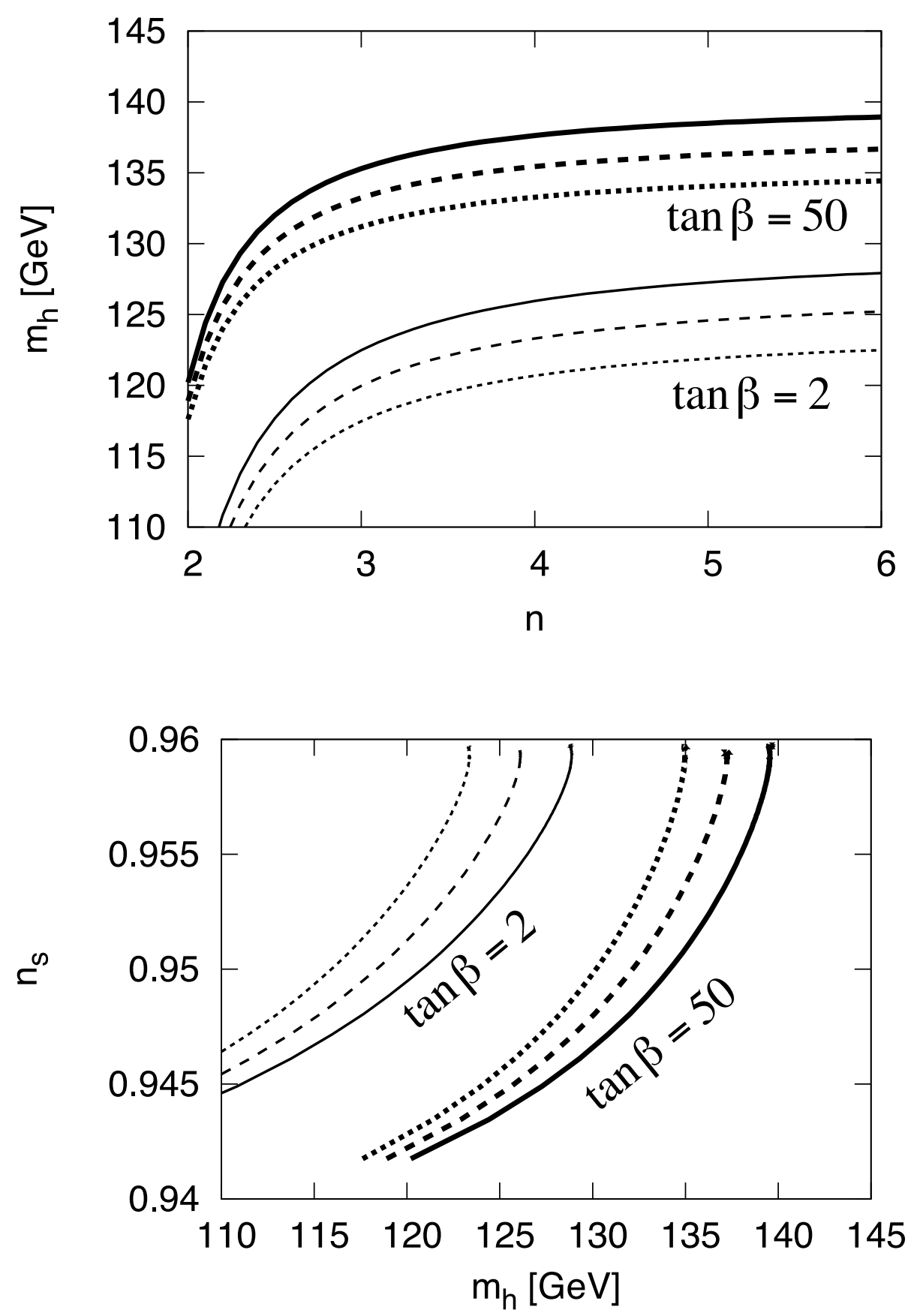

Figure 2: Same as Fig. 1, but for the case where all SUSY particle mass scales are set to be $\tilde{m}$. 
all the scalars and SSM gauginos have comparable masses of PeV. Then, if the lightest SUSY particle (LSP) is the Bino-like neutralino, a small amount of the R-parity violation may be needed in order to avoid the overproduction of the LSP. In this case the prime candidate for dark matter will be the QCD axion [19, 20].

The inflation scenario we consider is the new inflation model [21], and its energy scale is so low that only a negligible amount of the tensor mode is generated. Also non-Gaussianity of density perturbation as well as the running of the spectral index is negligibly small.

In summary, in this letter we have shown that the standard-model Higgs boson mass and the spectral index of density perturbation, which are seemingly totally independent of each other, are actually related in the inflation scenario using the B-L Higgs boson as the inflaton. This non-trivial relation will be soon checked by the LHC experiment and the Planck satellite.

\section{Acknowledgment}

K.N. would like to thank M. Ibe, T. Moroi and T. T. Yanagida for important comments and discussion. This work was supported by the Grant-in-Aid for Scientific Research on Innovative Areas (No. 21111006) [KN and FT], Scientific Research (A) (No. 22244030 [KN and FT] and No.21244033 [FT]), and JSPS Grant-in-Aid for Young Scientists (B)

(No. 21740160) [FT]. This work was also supported by World Premier International Center Initiative (WPI Program), MEXT, Japan.

\section{References}

[1] A. H. Guth, Phys. Rev. D23, 347-356 (1981); A. A. Starobinsky, Phys. Lett. B 91 (1980) 99; K. Sato, Mon. Not. Roy. Astron. Soc. 195, 467-479 (1981).

[2] K. Nakayama and F. Takahashi, JCAP 1110, 033 (2011) arXiv:1108.0070 [hep-ph]].

[3] T. Yanagida, in Proceedings of the "Workshop on the Unified Theory and the Baryon Number in the universe", Tsukuba, Japan, Feb. 13-14, 1979, edited by O. Sawada and A. Sugamoto, KEK report KEK-79-18, p. 95, and "Horizontal Symmetry And Masses 
Of Neutrinos" , Prog. Theor. Phys. 64 (1980) 1103; M. Gell-Mann, P. Ramond and R. Slansky, in "Supergravity" (North-Holland, Amsterdam, 1979) eds. D. Z. Freedom and P. van Nieuwenhuizen, Print-80-0576 (CERN); see also P. Minkowski, Phys. Lett. B 67, 421 (1977).

[4] G. R. Dvali, Q. Shafi and R. K. Schaefer, Phys. Rev. Lett. 73, 1886 (1994) [hep-ph/9406319]; G. Lazarides, R. K. Schaefer and Q. Shafi, Phys. Rev. D 56, 1324 (1997) [hep-ph/9608256]; G. R. Dvali, G. Lazarides and Q. Shafi, Phys. Lett. B 424, 259 (1998) hep-ph/9710314; R. Jeannerot, S. Khalil, G. Lazarides and Q. Shafi, JHEP 0010, 012 (2000) hep-ph/0002151]; W. Buchmuller, L. Covi and D. Delepine, Phys. Lett. B 491, 183 (2000) hep-ph/0006168; R. Jeannerot, J. Rocher and M. Sakellariadou, Phys. Rev. D 68, 103514 (2003) hep-ph/0308134]; V. N. Senoguz and Q. Shafi, Phys. Rev. D 71, 043514 (2005) hep-ph/0412102]; R. Jeannerot and M. Postma, JHEP 0505, 071 (2005) hep-ph/0503146]; V. N. Senoguz and Q. Shafi, hep-ph/0512170, M. ur Rehman, V. N. Senoguz and Q. Shafi, Phys. Rev. D 75, 043522 (2007) hep-ph/0612023]; K. Nakayama, F. Takahashi and T. T. Yanagida, JCAP 1012, 010 (2010) [arXiv:1007.5152 [hep-ph]]; S. Khalil, M. U. Rehman, Q. Shafi and E. A. Zaakouk, Phys. Rev. D 83, 063522 (2011) arXiv:1010.3657 [hep$\mathrm{ph}]]$.

[5] S. R. Coleman, E. J. Weinberg, Phys. Rev. D7, 1888-1910 (1973).

[6] J. R. Ellis, D. V. Nanopoulos, K. A. Olive, K. Tamvakis, Phys. Lett. B118, 335 (1982); Nucl. Phys. B221, 524 (1983).

[7] S. Weinberg, Phys. Rev. Lett. 59 (1987) 2607.

[8] E. Komatsu et al. [WMAP Collaboration], Astrophys. J. Suppl. 192 (2011) 18 arXiv:1001.4538 [astro-ph.CO]].

[9] G. Lazarides and Q. Shafi, Phys. Lett. B 258, 305 (1991).

[10] T. Asaka, K. Hamaguchi, M. Kawasaki, T. Yanagida, Phys. Lett. B464, 12-18 (1999). hep-ph/9906366; Phys. Rev. D61, 083512 (2000). hep-ph/9907559.

[11] V. N. Senoguz and Q. Shafi, Phys. Lett. B 582, 6 (2004) hep-ph/0309134; Phys. Lett. B 596, 8 (2004) hep-ph/0403294. 
[12] A. A. Starobinsky, Phys. Lett. B 117 (1982) 175.

[13] N. Arkani-Hamed, S. Dimopoulos, JHEP 0506, 073 (2005). hep-th/0405159;

G. F. Giudice, A. Romanino, Nucl. Phys. B699, 65-89 (2004). [hep-ph/0406088].

[14] M. Binger, Phys. Rev. D73, 095001 (2006). [hep-ph/0408240].

[15] M. Kawasaki, F. Takahashi, T. T. Yanagida, Phys. Lett. B638, 8-12 (2006). hep-ph/0603265]; Phys. Rev. D74, 043519 (2006). hep-ph/0605297]; T. Asaka, S. Nakamura, M. Yamaguchi, Phys. Rev. D74, 023520 (2006). hep-ph/0604132]; M. Endo, F. Takahashi, T. T. Yanagida, Phys. Lett. B658, 236-240 (2008). hep-ph/0701042]; Phys. Rev. D76, 083509 (2007). [arXiv:0706.0986 [hep-ph]].

[16] D. S. M. Alves, E. Izaguirre, J. G. Wacker, arXiv:1108.3390 [hep-ph]].

[17] L. Randall, R. Sundrum, Nucl. Phys. B557, 79-118 (1999). hep-th/9810155); G. F. Giudice, M. A. Luty, H. Murayama, R. Rattazzi, JHEP 9812, 027 (1998). hep-ph/9810442].

[18] J. Hisano, S. Matsumoto, M. Nagai, O. Saito and M. Senami, Phys. Lett. B 646, 34 (2007) arXiv:hep-ph/0610249.

[19] R. D. Peccei, H. R. Quinn, Phys. Rev. Lett. 38, 1440-1443 (1977).

[20] For a review, see J. E. Kim, Phys. Rept. 150, 1-177 (1987).

[21] A. D. Linde, Phys. Lett. B 108 (1982) 389. 